\title{
The Change of Chinese Research on Inclusive Education and Further Direction - What Can We Learn from Developed Areas
}

\author{
Yuantao Huang, Mei Zhang \\ East Asian Studies \\ University of Sheffield
}

\begin{abstract}
During decades, inclusive education has been accepted and developed in many countries. As a country with the largest number of populations, China also lays emphasis on inclusion in education. This paper concentrates on its development in China through comparing the existing studies and interviews with experts by using the $U K$ as an example of developed countries. As a result, the characters of Chinese research on inclusive education are found in educational values, theoretical and practical considerations. Based on those features and the progress of developed countries, like the UK, this paper also provides two main potential directions, including theoretical and practical aspects, to Chinese inclusive education for future research. Both concerns can not only benefit the relevant research in China but also inspire other places to rethink their research points.
\end{abstract}

\section{Introduction}

Nowadays, discrimination and exclusion are existing in society, which can be treated as the problems of equity, including gender, races, education, language, religion, and so on. As the brief introduction in the last section, inclusive education attempts to resolve the inequity phenomena in education. Certainly, the different countries have their specific issues need to be solved. For example, the UK and USA consider education to overseas immigrants, but China pays more attention to the inner migrants, which largely depends on the stage of social development and local conditions. Having an education is a fundamental human right and whether the Education for All (EFA) or inclusive education has already been the new tendency in the educational field [1]. Unfortunately, the discriminatory and exclusive phenomena still exist in the majority of schools, although there is plenty of theoretical and practical research on inclusive education so far.

However, such studies, particularly in the early stage, prefer to investigate the individuals with SENs and overlook some other groups people in disadvantaged situations. This phenomenon is likely because the dominant understanding of inclusive education is initially linked to deal with the educational issues for the disabled learners in some countries [2][3]. Oppositely, according to the famous British professor, Tony Booth and Mel Ainscow, who considering inclusive education broadly, inclusion in education is considering the welcoming environment and respecting the diversity of all learners, which is not simply about the children with impairments [4].

Although the term, inclusive education raised from the western, it has been accepted and adopted by different areas around the world gradually, such as some Arabic countries, China, Japan, South Korea [5][6][7][8]. As a typical eastern country, China has a fairly long history of education with its unique characters that may still have a far-reaching influence on the current educational system. Therefore, some similar ideas of inclusive education can be found in history like 'You jiao wu lei,' and 'Yin cai shi jiao.' Both views emphasize the education should be for all no matter who they are and what they do, which could be related to the inclusive education that highlights to solve the exclusion and respect diversity in education. Interestingly, a similar idea of inclusive education in history does not make it be understood broadly in Chinese research. During the period of learning experience from developed countries, China has combined local characteristics with the perspective of inclusive education to put it into practice, called 'Learning in the regular classroom' (LRC). It is translated from the Chinese 'Sui ban jiu du,' which is a practical model of teaching disabled students in traditional schools [9][10]. Although plenty of articles focus on educational equity, like gender discrimination, urban-rural distinction, migrants' children, and teacher-student relationships, these topics are rarely linked to inclusive education. It reveals the development of inclusive education in China is insufficient particularly lack a guide to promote. Thus, this research will focus on comparing and contrasting inclusive education between Chinese research and British articles to evaluate the differences and similarities and to further provide 
suggestions to Chinese studies, which could also inspire other researchers to reconsider their research directions.

Overall, this paper aims to explore the changes in academic research on inclusive education, particularly the Chinese studies, and compared with the UK, to further consider potential aspects of improving inclusive education studies in China. Therefore, there are three main research questions: (1) What are the differences and similarities between Chinese and British research on inclusive education?

(2) What are the situations of Chinese inclusive education studies?

(3) What can Chinese inclusive education learn from the developed regions like the UK?

\section{Method}

In order to achieve the answer to each research question, this paper will primarily review the existing articles related to both Chinese and British inclusive education. It is because comparing previous studies can evaluate what has been done and what has not in China and the UK, which is a significant approach to figure out the differences and similarities. The previous studies will be found on the academic database Google Scholar, CNKI, the most prominent academic database in China and Star plus, the University's library system, which can ensure the quality of the studies. All the literature is searched by using inclusive education, inclusion in education, China, Chinese, the UK, Britain and British as the keywords to ensure that all chosen articles are relevant to the research questions.

Except for critically reviewing the literature, this paper will also conduct interviews with the Chinese and British experts in this research field, which intends to deeply investigate the present situation of research on inclusive education and its practice. It can be more direct to reflect the differences and similarities between both countries than only read other's studies; therefore, combing the literature and interviews together will fulfil all the research questions. The questions employed in the interviews are fully discussed with the supervisor to make sure they can link to those three research questions. According to the period of searching literature, several experts in China and the UK, who are mentioned many times in relevant studies and make contributions to this field, are selected as the potential participants, and after communicating with most of them, two of them willing to accept the further interviews.

The experts are from Beijing Normal University and the University of Cambridge; also, they have the academic prestige of inclusive education studies. The details of these candidates will be in Table 1 below.

Table 1. Experts' information

\begin{tabular}{|l|l|l|l|l|}
\hline Number & Gender & Nationality & Subject & $\begin{array}{l}\text { First } \\
\text { time } \\
\text { to } \\
\text { know } \\
\text { IE }\end{array}$ \\
\hline 1 & M & Chinese & $\begin{array}{l}\text { Special } \\
\text { Education }\end{array}$ & 1990 s \\
\hline 2 & M & British & $\begin{array}{l}\text { Psychology } \\
\text { and } \\
\text { Philosophy }\end{array}$ & N/A \\
\hline
\end{tabular}

(Source: interview)

This table presents the expert, from China, have twenty years experiences of learning and working on inclusive education, although his original academic research field is special education. Another expert, from Britain, also has rich experience of inclusive education, and his works have influences on Chinese and even the world's research of inclusive education.

\section{Critical literature review and interviews}

The global trend of studying on inclusive education seems to link to special education closely, and according to Messiou's research in 2017, only eight per cent articles, in the International Journal of Inclusive education from 2005 to 2015, were relevant to the broad perspective of inclusive education or the diversity in education [11].

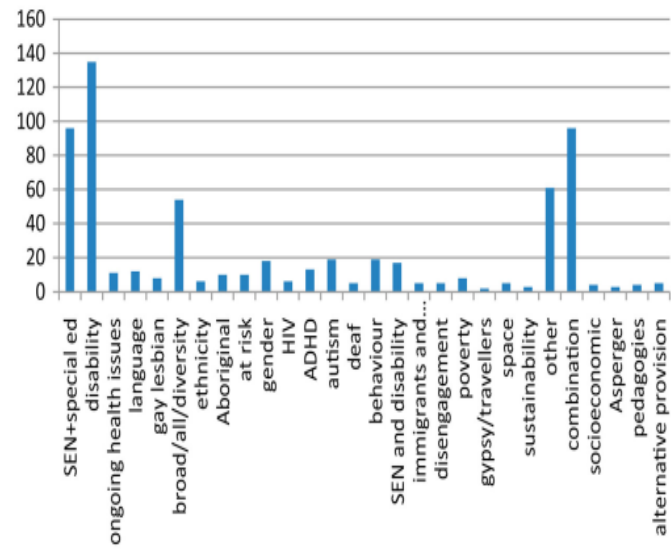

Figure 1 . The ten years' topics in the International Journal of Inclusive Education, adopted from Messiou, 2017).

Based on Figure 1, it likely reflects that although the term 'inclusion' is related to all aspects in society, in educational research, it is only or generally associated to special education, special educational needs or disability. Therefore, 
it is easy to find that the narrow understanding of inclusive education is dominant in research, which can be proved by both Chinese and British experts through the interviews.

\subsection{Inclusive education in the UK}

This section will mainly review the research from theoretical and practical parts. Of course, the British studies on inclusive education are generally following the global trend that many articles only concern children with disabilities in education, which is basically about how to understand inclusive education. According to the interview, the British professor assumes that the debates of understanding inclusive education is still existing after it emerged in 1994. As same as many concepts, the understandings of inclusive education are various and just can be divided as broad and narrow, which mainly discusses whether all students or specific groups. Although there are different understandings of inclusive education, everyone has an educational right should be admitted, which should be fundamental in education.

In general, the broad understanding of inclusive education stresses education should fulfil all students' needs, but the narrow one pays more attention to disabled groups. According to Lipsky and Gartner and Messiou's studies, the disabled study is always adopted as the example to analyse inclusive education, which, as aforementioned, is an international viewpoint [11][12]. On the other side, Ainscow and Booth propose that inclusive education should not only face the students with special educational needs but also all students [13]. In the meantime, Booth claims the inclusive education as a dynamic process rather than a statement, which is mainly to increase students' participation and reduce educational exclusion. He highlights that students should participate in learning, community, and culture; meanwhile, the exclusive phenomena in education is unacceptable [13]. Hodkinson illustrates "inclusion must be a broad church with solid foundations where exclusion from society is accepted as having a common basis in "intolerance to difference"' [14]. Thus, from this broad perspective, inclusion refers to special educational needs, race, culture, social positions, age, ethnicity, gender, and sexual orientation.

Of course, it cannot judge which understanding is right or wrong because the researchers stay at the different positions. For example, Ainscow and Cesar list five ordinary senses of inclusive education in research, including both narrow and broad ways [2]. No matter which ways of researchers' understanding of inclusive education, everyone admits that education is one of the fundamental human rights, which means every individual should have equal opportunity to receive a high-quality education. Equality is an unavoidable topic, no matter which way of considering inclusive education are the researchers following, which links to the human right. For example, in 2009, the UNESCO's definition of inclusive education mentions that every child has rights to accept education in the regular educational system. It means if a child is segregated from the regular school because he or she was categorised as 'disability,' it will be treated as violating human rights, which is a severe issue like gender discrimination and racism. However, Farrell demonstrates that inclusive education would have issues if the studies only consider human rights [15]. In fact, many years ago, British researchers noticed that simply enrolling students with learning difficulties may not be a good choice. Runswickcole points that most children with special educational needs in mainstream school report that there are no such respect and understandings [16]. As the British professor during his interview suggests, inclusive education, in reality, is still following the narrow perspective though more academic studies begin to accept the broad theory. To fix the issues in practice, Booth and Ainscow have been established an index for inclusion since 2002 to guide school developing an inclusive values and environment. So far, the index has already been translated and adopted by many developed and developing countries, which could be seen as an early and mature action of inclusion in education.

Overall, inclusive education has attracted researchers' attention since 1994, but the mainstream studies largely depend on Western values. The British professor comments, in the interview, that every culture has conflicts that make the inclusive education different under their system.

\subsection{Inclusive education in China}

Chinese research on inclusion in education is also following the global trend that closely links to special education, but it has different characters. China's inclusive education study primarily focus on its development history, other countries' research, and Chinese situation, including its theory and practice [10][11]. Although Chinese researchers argue that the understanding of inclusive education as an essential topic, it is different from the UK because the core consideration is about 'learning in the regular classroom' in both theoretical and practical studies. It makes many scholars misunderstand that LRC is same as inclusive education, and even directly mixed them up in their research [17]. This issue has changed a bit but still exist, according to 
the Chinese professor who involved in the interview, and he supposes that there are still misunderstandings of inclusive education in China, today. He provides a typical example that the Chinese government believes developing LRC is doing inclusive education, which makes people misunderstand they are the same.

Of course, many Chinese scholars can distinguish these two terms, except for the professor in the interview, such as professor Huang, Deng, and Li. Some of them are changed their mind with the development of inclusive education, which definitely accelerates its progress in Chinese research. In the early studies, professor Huang and Deng summarise many research and ideas of inclusive education from British articles, and they introduce a broad perspective in their studies [18]. Although they can recognise the differences between broad and narrow views of inclusive education, their words and examples may mislead readers. For instances, Deng and McBryan demonstrate that 'if we stand by the broad perspective of inclusive education, we could neglect the tiny differences among inclusion, integration, and mainstreaming. Thus, we can treat all attempts, placing children with special education needs in ordinary schools fully or selectively, as inclusive education [18].'

Talking about education in China cannot avoid the far-reaching influence of Confucian thoughts. As aforementioned, inclusive education seems to underpin the western value mainly, and may not be entirely appropriate to other cultures. Therefore, Chinese scholars concern education for all or equality in education prefer the traditional perspective of education, like 'You jiao wu lei,' and 'Yin cai shi jiao.' Both terms are similar to education for all and respect diversity in education, which provides the possibility of developing inclusive education in Chinese education. Therefore, considering the differences cultural background Chinese research like Deng and Jing raised an idea of localising the term, inclusive education [19]. Deng and Su believe that so far, the relevant studies on inclusive education spend too many concerns on individual's rights, needs, and equality, but neglect the respect toward different background [20]. Although localisation would spend a long period to achieve an appropriate explanation of inclusive education for Chinese education, this period can also be a process that supplements inclusive education itself by standing at a different cultural position.

Overall, inclusive education has been studied and practised for many years and gained some impressive achievements. For example, the Chinese expert in his interview emphasises that the practical model, LRC, increases the enrolment of children with disabilities in regular schools, which literally provides an excellent learning choice to them. He claims that LRC model dramatically promotes the enrolment rate of children with disabilities, in particularly in the early years. However, its development is restricted by Chinese educational environment and the global trend. The Chinese expert further argues that no matter the researchers, schools and even the government initially concerns the rates, and they barely spend time on the quality of education for those who need additional learning needs. Therefore, it can be found easily that in schools, most in-service teachers do not have sufficient experience and skills to cultivate those students and the support for teachers are also limited.

\section{Discussion}

Through reviewing academic articles and conducting interviews with experts, many distinctive characters of inclusive education between China and the UK could be found in both studies on the theory and practice aspects. For the similarities, there are three main points, including both theoretical and practical levels. Firstly, in recent years, many researchers accept the broad understanding of inclusive education in theory gradually. However, secondly, the narrow understanding of it is dominated in practical level, which still primarily focus on the students with disabilities. Thirdly, inclusive education is difficult to act into teaching whether the broad or narrow perspective under the current conditions. Except for these three main similarities, the values behind inclusive education are also partially the same. As aforementioned, inclusive education based upon the western values of human rights and equality, but differently, Chinese research prefers to relate Confucian thoughts. The reason for partially the same is because the Chinese values, 'You jiao wu lei' and 'Y in cai shi jiao,' are similar to education for all and respect the diversity in education. On the other side, the practice of inclusive education in the UK is more mature than China because British schools seem to have a systematic support network. Of course, the objective conditions are also differentiable, such as the class scale, training program, students' background, and some other regional characteristics.

According to the review of Chinese research on inclusive education, it can be found several popular themes at the earlier stage, including the discussion and introduction of inclusive education, the comparison of inclusive education and special education. Then, many researchers change their concerns from the theoretical level to the practical side, and some research on inclusive education begin to evaluate the experience of 
developed countries and the LRC model in schools. It has a far-reaching influence on Chinese inclusive education studies because this model seems to be an essential concern of Chinese research on inclusive education, which closely relates to special education or disability study. It makes many Chinese studies on inclusive education cannot get rid of the influence of special education, and even misleads some researchers to directly use LRC to replace inclusive education in their research. In recent years, some scholars begin to rethink the situation of inclusive education in China and propose that the term inclusive education needs to be localised with Chinese conditions and culture. It makes relevant research pay attention to the practical detail, and in-service teachers' opinions and attitudes on inclusive education attract attention gradually.

Compared with the development of inclusion in the UK, Chinese academic field still has room for improvement. To increase the quality of inclusive education, Britain has built the relevant network, including schools, community, university, and local authorities. It, indeed, provides strong support to the development of inclusive education in both academic research and practice. Moreover, there is an index for inclusion in the UK, which has been translated into many different languages. Also, it is applied widely in both developed areas like the USA, Australia, many European countries, and some developing regions. It can be treated as a guide for schools to practise inclusive education comprehensively through considering the perspectives of government, teachers, families and society. By contrast, the cooperation among such sectors is limited in Chinese research on inclusive education, especially the support from the community, which reflects exploring the positive effect of community in education can be a potential research direction of inclusive education. In addition, Chinese research on inclusive education lack of consideration of evaluating the other stakeholders' opinions, such as students, students' parents, and in-service teachers, which has been mentioned by several scholars but still needs to be improved. Most importantly, although China studies on inclusive education for decades, there is no standard or guide to assess and develop its implementation. Thus, the British index may be an excellent example of Chinese inclusive education research in the future.

\section{Conclusion}

In summary, this paper main reveals the research situation of inclusive education in China by evaluating academic articles. According to the research, although inclusive education has been improved for many years in China, it is still staying at an early stage and mixed with special education. Compared with the UK, inclusive education in China lacks a systematic structure, including understandings, practice, and support. Therefore, further studies could pay more attention to establish a systematic framework of developing inclusive education under Chinese educational conditions.

\section{Reference}

[1] Miles, S., \& Singal, N. (2010). The Education for All and inclusive education debate: conflict, contradiction or opportunity? International Journal of Inclusive Education, 14(1), 1-15.

[2] Ainscow, M., \& Cesar, M. (2006). Inclusive education ten years after Salamanca: Setting the agenda. European Journal of Psychology of Education, 21(3), 231-238.

[3] Thomazet, S. (2009). From integration to inclusive education: does changing the terms improve practice? International Journal of Inclusive Education, 13(6), 553-563.

[4] Booth, T. \& Ainscow, M. (2016). The Index for Inclusion. 4th ed. Cambridge: Index for Inclusion Network.

[5] Alborno, N., \& Gaad, E. (2014). 'Index for Inclusion': A Framework for School Review in the United Arab Emirates. British Journal of Special Education, 41(3), 231-248.

[6] Lei, J.H. \& Deng, M. (2007). On several relations in the process of developing inclusive education. Chinese Education and Society, 40(4), 33-43.

[7] Futaba, Y. (2016). Inclusive Education under collectivistic culture. Journal of Research in Special Educational Needs, 16(1), 649-652.

[8] Kim, Y. (2014). Inclusive education in South Korea. International Journal of Inclusive Education, 18(10), 979-990.

[9] Chen, Y.M., \& Wang, T.Z. (2015). A visualization analysis of the research on domestic inclusive education. A Journal of Modern Special Education (Higher Education), (6), 7-11.

[10] Du, Z.Q. \& Zhi, S.R. (2015). Hotspot domains and frontier topics of Chinese special education: Based on knowledge mapping of keywords of 'Chinese Journal of Special Education' published in recent ten years. Education Research Monthly, (2), 18-23.

[11] Messiou, K. (2017). Research in the field of inclusive education: time for a rethink? International Journal of Inclusive Education, 21(2), 146-159.

[12] Lipsky, D., \& Gartner, A. (1998). International 
perspectives on special education reform. European Journal of Special Needs Education, 13(1), 128-133.

[13] Ainscow, M., \& Booth, T. (1998). From them to us: An international study of inclusion in education. London: Routledge.

[14] Hodkinson, A. (2011). Inclusion: A Defining Definition? Power and Education, 3(2), 179-185.

[15] Farrell, P. (2000). The impact of research on developments in inclusive education. International Journal of inclusive education, 42(2), 153-162.

[16] Runswick-Cole, K. (2011). Time to end the bias towards inclusive education? British Journal of Special Education, 38(3), 112-119.

[17] Li, L. (2009). Some issues in current research of Learning in Regular Classroom. Chinese Journal of Special Education, 113(11), 3-7.

[18] Deng, M. \& Poon-McBrayer, K. (2003). Review of the literature on inclusive education and its implication for Chinese special education, Chinese Journal of Special Education (Bimonthly), (4), 1-7.

[19] Deng, M., \& Jing, S. (2013). From Learning in Regular Classrooms to Equal Regular Education: Reflections on the Localization of Inclusive Education in China. Chinese Journal of Special Education, 158(8), 3-9.

[20] Deng, M. \& Su, H. (2012). 'Grafting' and 'Regeneration' of integrated education in China: A socio-cultural analysis. Journal of Educational Studies, 8(1), 83-89. 